Ann. Abeille, I965, 8 (4), 27I-283.

\title{
ÉTUDE BIOMÉTRIQUE DE QUELQUES CARACTÈRES MORPHOLOGIQUES DE L'ABEILLE NOIRE FRANÇAISE (APIS MELLIFICA MELLIFICA)
}

\author{
J. FRESNAYE \\ Station expérimentale d'Apiculture, \\ Centre de Recherches agronomiques du Sud-Est, Montfavet (Vaucluse)
}

SOMMAIRE

Dans cette étude l'auteur définit les caractéristiques biométriques moyennes de l'abeille indigène française (Apis mellifica mellifica). La couleur est noire, avec parfois de petites taches jaunes sur le $\mathbf{~ e r ~ t e r g i t e ~ a b d o m i n a l , ~ l ' i n d e x ~ c u b i t a l ~ e s t ~ d e ~} 1,70$, la pilosité sur le $5^{\mathbf{e}}$ tergite de $0,46 \mathrm{~mm}$, l'index du tomentum de $0,9 \mathrm{I}$, la longueur de la langue de $6,35 \mathrm{~mm}$. On met également en évidence les difficultés de diagnostic dans les analyses biométriques de certaines colonies hybrides.

\section{INTRODUCTION}

La détermination précise des différentes races d'abeilles et de leurs hybrides est essentielle dans un programme de sélection moderne. Elle peut être obtenue à l'aide des nombreux caractères morphologiques utilisés en systématique mais le temps nécessaire à la totalité des mesures possibles dépasserait les moyens pratiques du sélectionneur et de l'éleveur. Nous n'avons donc considéré la biométrie que comme une méthode de travail qui permet de mettre en évidence la pureté de race ou, au contraire, le caractère hybride des colonies sélectionnées. Il convient donc de limiter le nombre des caractères indispensables à mesurer pour obtenir des analyses satisfaisantes et de les choisir en fonction de certains critères.

Tout d'abord, il faut que ces caractères soient d'une utilisation suffisamment généralisée pour permettre des comparaisons nombreuses avec les analyses biométriques pratiquées antérieurement mais qui n'ont que très peu porté sur l'abeille française, laquelle peut être encore considérée comme mal connue. La variation de chaque caractère doit être suffisamment importante d'une race à l'autre pour per- 
mettre une détermination certaine. Les mesures doivent être faciles et rapides car l'éleveur ne peut généralement consacrer qu'une faible partie de son temps aux analyses biométriques. Enfin, il faut éviter autant que possible les données subjectives dont l'appréciation est sujette à variations d'un utilisateur à l'autre ou même à différentes périodes pour un même utilisateur.

Un grand nombre de recherches concernant la biométrie de l'abeille ont été effectuées par GoETzE depuis I926. L'essentiel des travaux de cet auteur et tout ce qui concerne la biométrie de l'abeille est rassemblé dans une publication récente (GoETzE, I963) ; nous n'y reviendrons donc pas. Nous avons limité, en ce qui nous concerne, le nombre des caractères morphologiques étudiés à ceux qui ont été retenus par RuTTNer et MAckensen (I954) ainsi que par RuTTNer (I963), c'est-à-dire :

I. La couleur.

2. L'index cubital.

3. La pilosité du $5^{\text {e }}$ tergite abdominal.

4. La largeur du tomentum sur le $4^{\mathrm{e}}$ tergite.

5. La longueur de la langue.

Les mesures de l'index cubital se prêtent à la construction d'histogrammes qui permettent de déceler des hybridations, ainsi qu'au calcul d'un écart-type ( $\sigma$ ) dont la valeur est également influencée par les hybridations et par la consanguinité. La translation discoïdale a été décrite par GoETzE. Elle a été étudiée en ce qui concerne l'abeille noire française par LouIs (I963). Nous ne l'avons pas incluse dans nos analyses car les mesures sont assez longues et délicates à pratiquer et les interprétations statistiques sont difficiles. L'ensemble des mesures de nos analyses porte uniquement sur les ouvrières. Les mâles étant parthénogénétiques, leur étude morphologique ne fournit aucun renseignement sur les géniteurs mâles qui ont participé à la fécondation de la reine. Ėn outre, l'époque à laquelle l'éleveur dispose de suffisamment de temps pour les analyses biométriques se situe en fin de saison apicole, juste avant l'hivernage; à ce moment les mâles ont disparu presque totalement dans les colonies.

Les analyses que nous avons effectuées nous permettent de définir les normes moyennes et les limites extrêmes de l'abeille noire française pour chacun des caractères morphologiques mesurés.

\section{MATÉRIEL ET MÉTHODE}

\section{Prise des échantillons}

Les échantillons d'abeilles destinés aux analyses biométriques sont prélevés dans les ruches de la Station d'Apiculture expérimentale. Un cadre couvert d'abeilles est secoué au-dessus d'une boîte ou d'une cagette type Liebefeld. roo ouvrières sont nécessaires à l'analyse. Les échantillons sont apportés au laboratoire. Les abeilles qui ne peuvent être examinées le jour même reçoivent de l'eau et du sucre candi. Les abeilles sont tuées à l'aide d'un morceau de coton hydrophile imbibé d'éther acétique placé dans la cagette. Ce coton doit être introduit au moins vingt minutes avant le début de l'analyse faute de quoi les abeilles reprennent vie et essaient de voler ou de piquer. Avant de commencer une analyse, on place habituellement le coton imbibé d'éther dans la cagette suivante. 


\section{Mesures biométriques}

a) Couleur de l'exosquelette.

Le caractère est estimé, principalement sur le premier tergite abdominal. Il s'agit de déterminer la présence, ou l'absence, ainsi que l'importance des taches jaunes. Nous utilisons une échelle de 4 classes:

\begin{tabular}{|c|c|c|}
\hline Classes & Caracteres & Interprétation \\
\hline 1 & $\begin{array}{l}\text { Coloration noire lotale de tous les segments } \\
\text { abdominaux. On ne la trouve qu'assez excep- } \\
\text { tionnellement. }\end{array}$ & Abeille noire \\
\hline 2 & $\begin{array}{l}\text { Coloration noire aver de très petites taches } \\
\text { jaunes de chaque coté du premier tergite } \\
\text { abdominal. }\end{array}$ & Abeille noire \\
\hline 3 & $\begin{array}{l}\text { Coloration jaune sous forme de } 2 \text { grandes } \\
\text { taches, pouvant aller jusqu'à couvrir la quasi- } \\
\text { totalité du premier segment abdominal. }\end{array}$ & Abeille hybride \\
\hline 4 & $\begin{array}{l}\text { Coloration jaune ou orangée du } \text { der }^{\text {or }} \text { des } 2 \\
\text { et } 3 \text { premiers anneaux de l'abdomen. }\end{array}$ & $\begin{array}{l}\text { Abeille étrangùre } \\
\text { à la race noire }\end{array}$ \\
\hline
\end{tabular}

L'estimation est faite sur les roo abeilles de l'échantillon.

b) L'index cubital.

L'index cubital est l'un des caractères les plus précis dont on dispose actuellement en biomét rie. Les mesures portent sur les Ioo abeilles de l'échantillon. Pour le calculer on mesure les deux portions de nervure formant un angle obtus à la base de la troisième cellule cubitale, et l'on établit le rapport $a / b$ (fig. I).

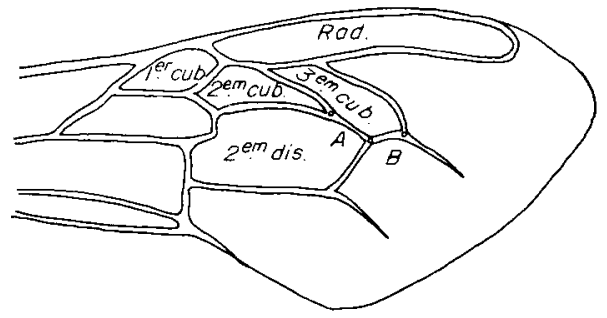

FIG. I. - Hile antérieure droite d'abeille onvière Apis mellifica mellitica L'index cubital se calcule en faisant le rapport entre les portions $A$ et $B$ des nervures inférieures de la $3^{\theta}$ cellule cubitale

Les ailes antérieures, (une par abeille et toujours le même côté) sont montées entre deux lames de verre mince de 180 millimètres de long par 45 millimètres de large environ. Les ailes sont d'abord arrachées du thorax à l'aide d'une pince à dissection puis jetées dans de l'alcool à $70^{\circ}$ légèrement sucré. Elles sont ensuite placées sur la première lame, sur deux rangs, toujours dans le même sens et bien en ligne, de façon à pouvoir passer rapidement de l'une à l'autre lors des mesures. Lorsque l'alcool est évaporé les ailes se trouvent légèrement fixées à la lame par le sucre. On couvre alors avec la seconde lame et un ruban adhésif placé aux 4 coins fixe l'ensemble. En procédant ainsi, les mesures peuvent être faites longtemps après le montage des ailes ce qui permet l'étalement du travail. 
Les mesures sont notablement facilitées par l'utilisation du dispositif décrit par RutTNER (ig63). Il consiste en un film dont les graduations micrométriques sont superposables aux deux portions de nervures à mesurer. Ce film est placé dans l'oculaire de la loupe ; un grossissement $\times 4^{\circ}$ permet des mesures rapides et précises.

Pour permettre la construction d'un histogramme présentant une distribution normale de GaUSS, les rapports sont regroupés dans une série de classes, distribuées selon une progression logarithmique. Plusieurs distributions de ce type ont été établies, notamment par GoETZE (I930 et I96I) et RutTner ( 1963 ). Nous avons conservé cependant celle de Dreher (I950) qui est utilisée dans notre laboratoire depuis de nombreuses années et nous permet la comparaison directe des résultats obtenus dans toutes les régions.

Les classes de DREHER se répartissent de la façon suivante :

\begin{tabular}{|c|c|c|c|c|c|c|c|c|c|c|c|c|c|c|c|}
\hline $\begin{array}{l}\text { Classe } \\
\text { Index }\end{array}$ & 0,667 & 1 & 0,724 & 2 & 0,786 & 3 & 0,852 & $\begin{array}{l}4 \\
--\end{array}$ & $0,9 \geq 3$ & 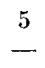 & 1,00 & $\begin{array}{c}6 \\
-\end{array}$ & 1,08 & & \\
\hline Classe & & 7 & & 8 & & 9 & & 10 & & 11 & & 12 & & & \\
\hline Index & 1,08 & - & 1,17 & $\ldots$ & 1,27 & - & 1,38 & - & 1,50 & - & 1,63 & - & 1,78 & & \\
\hline Classe & & 13 & & 14 & & 15 & & 16 & & 17 & & 18 & & & \\
\hline Index & 1,78 & - & $1,9^{\prime} \mathbf{t}$ & - & 2,13 & - & 2,33 & - & 2,57 & - & 2,85 & - & 3,17 & & \\
\hline Classe & & 19 & & 20 & & 21 & & 22 & & 23 & & 24 & & 25 & \\
\hline Index & $3, \mathbf{1 7}$ & --- & 3,55 & - & $\{, 00$ & - & 4,56 & - & 5,25 & - & $6,1^{\prime}$ & - & 7,33 & $\ldots$ & 9,00 \\
\hline
\end{tabular}

Il n'est pas nécessaire de calculer individuellement chacun des index mesurés. L'utilisation d'une table qui donne immédiatement la classe correspondant à l'index à partir des deux mesures initiales, permet un gain de temps important. L'index cubital moyen de l'échantillon est alors calculé d'après l'index moyen de chaque classe. On peut aussi calculer la classe moyenne et faire la transformation en index réel. Dans l'un et l'autre cas la différence avec l'index moyen calculé à partir de tous les index est négligeable.

L'histogramme nous renseigne visuellement sur la répartition des index dans les classes, mais statistiquement il est inutilisable. Par contre, le calcul de l'écart-type $(\sigma)$ qui est avec la moyenne arithmétique le paramètre le plus efficace, nous permet d'obtenir de façon chiffrée l'expression de la répartition des index. Il est calculé d'après le nombre d'abeilles groupées dans chaque classe, suivant la formule classique :

$$
\sqrt{\frac{\mathrm{S}\left(x^{2}\right)-\mathrm{G}^{2} / n}{n-\mathrm{I}}}=\sigma \quad \text { (écart type) }
$$

dans laquelle nous avons:

$x^{2}=$ Carré du produit de l'index moyen de chaque classe par le nombre d'abeilles groupées dans chacune de ces classes.

$\mathrm{G}^{2}=$ Carré de la somme des produits des index moyens de chaque classe par le nombre d'abeilles groupées dans chacune de ces classes.

$n=$ Nombre total d'abeilles de l'échantillon.

L'écart-type ne remplace cependant pas totalement l'histogramme car il ne peut nous renseigner sur la forme de la courbe qui peut être unimodale ou bimodale, ce qui est très important dans les analyses.

\section{c) Pilosité du cinquiène tergite abdominal.}

Ce caractère est mesuré, en observant l'abeille de profil, sur le $5^{\mathrm{c}}$ tergite abdominal. Dans certaines méthodes on estime simplement ce caractère en le comparant à la largeur du I er article du tarse de la patte postérieure de l'abeille. On obtient alors trois catégories "plus court ", "égal "ou "plus long " que la largeur de l'organe de référence. Il nous a semblé que cette méthode était trop imprécise et subjective pour donner des renseignements utiles dans les cas d'hybridation. Nous l'avons remplacée par la mesure sous la loupe, munie d'un oculaire micrométrique avec un grossissement de $\times 40$ (fig. 2). La précision ainsi obtenue dépasse le dixième de millimètre. Les mesures portent sur to abeilles prises au hasard dans l'échantillon. 


\section{d) Largeur du tomentum.}

Ce caractère est mesuré sur le $4^{\mathrm{e}}$ tergite ou antepénultième tergite abdominal. Il est souvent exprimé par un index donné par le rapport existant entre la bande pileuse et la partie glabre de ce tergite. Nous l'avons remplacé par la mesure de la bande pileuse ce qui évite une partie des calculs. L'équivalence avec l'index correspondant s'établit comme suit :

$$
\begin{array}{ll}
\text { Largeur du tomentum.. } & 0,3-0,4-0,5-0,6-0,7-0,8-0,9-1,0-1,1 \\
\text { Index } \ldots \ldots \ldots \ldots \ldots & 0,27-0,40-0,55-0,75-1,00-1,33-1,80-2,50-3,66
\end{array}
$$

Les mesures sont faites sous la loupe munie d'un oculaire micrométrique avec grossissement $\times$ ro. Les mesures portent sur to abeilles prises au hasard dans l'échantillon. En dépit des mesures sous la loupe l'observation de ce caractère reste assez subjective car la lisière de la bande pileuse n'est pas nettement définie. Fn outre, on est influencé par la densité de la toison. Les mesures peuvent donc varier légèrement suivant les observations.

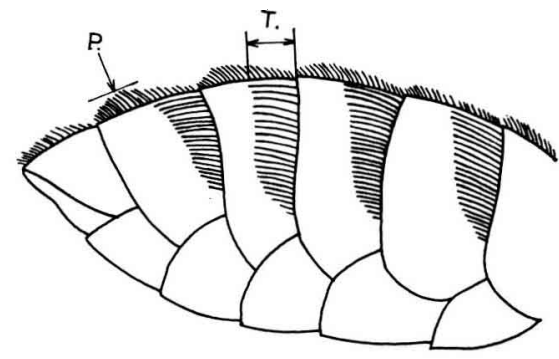

FIG. 2. -- Abdomen d'abeille ouvrière ApisImelfifica mellifica vu de profil

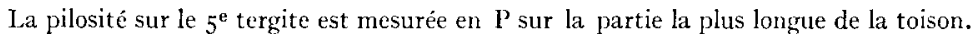
La largeur du tomentum est mesurée en T sur le 4 e tergite, mais lorsque l'abeille est vue sur le dos

\section{e) Longueur de la langue.}

Diverses méthodes ont été utilisées pour mesurer la longueur des langues d'abeilles. Plusieurs modèles de "glossomètres ", appareils contenant du sirop que les abeilles doivent vider jusqu'à la longueur maximum de leur langue, ont été essayés. Ces méthodes tombent actuellement en désuétude. Certains auteurs préconisent la projection de la langue sur un écran pour obtenir des mesures plus précises. La mesure directe, sous la loupe munie d'un oculaire micrométrique est satisfaisante tant au point de vue de la précision qu'à celui de la facilité de travail : la tête de l'abeille est tranchée, retournée et fixée à l'aide d'une aiguille entomologique. La glosse est alors mise en extension maximum en prenant soin de ne pas la détériorer. Les mesures portent sur le submentum, le mentum et la glosse. Elles sont faites sous grossissement $\times 16$, sur 10 abeilles.

\section{RÉSULTATS}

Depuis un certain nombre d'années l'habitude d'importer des reines de races étrangères s'est développée en Frrance. Les apiculteurs espèrent trouver dans ces abeilles des qualités de rendement, de prolificité et de douceur qu'ils estiment peu développées chez l'abeille indigène. L'abeille italienne qui jouit dans le monde d'une grande notoriété a également séduit l'apiculteur français. La proximité de son pays d'origine et le développement du commerce des reines dans ce pays ont contribué à l'importation massive de reines de race italienne dans beaucoup de nos régions. L'abeille carniolienne et l'abeille caucasienne sont également importées, plus ou moins sporadiquement. Enfin on peut signaler quelques cas d'importation d'abeilles cypriotes et d'abeilles d'Afrique du Nord. Ces apports de races étrangères sont la 
cause d'hybridations nombreuses. Il faut donc être particulièrement attentif et prudent dans les conclusions des analyses biométriques, aussi bien pour celles qui sont utilisées pour la sélection que pour celles qui permettront de définir les normes de l'abeille indigène française. Nous avons réalisé cette étude à 1'aide des analyses biométriques de ruches appartenant à notre Station. Les reines de ces ruches sont issues de souches déjà reconnues exemptes d'hybridation. Ce travail est complété par la comparaison avec quelques ruches hybrides spécialement préparées ainsi que quelques colonies de races étrangères. Ces dernières permettent de mettre en évidence les limites de sécurité des diagnostics établis pour chacune des ruches. 240 colonies au total, ont ainsi servi aux analyses biométriques de cette étude.

\section{a) La couleur}

La couleur qui est estimée, et non mesurée, n'a pu permettre la construction d'un histogramme détaillé. On trouve très peu de colonies dans la classe I (abeilles noires sans tache jaune). La classe 2 (abeilles noires avec de petites taches jaunes), réunit la majeure partie des colonies de race noire pure. Dans la classe 3 on trouve quelques colonies hybrides. Les abeilles de race italienne sont dans la classe 4 . La couleur de l'abdomen est le meilleur critère de discrimination entre la race noire et les races ayant un ou plusieurs anneaux jaunes à condition que les taches colorées soient suffisamment larges. L'apparition de taches jaunes importantes sur un grand nombre d'abeilles dans tne colonie est un signe indiscutable d'hybridation, mais qui ne permet pas à lui seul de définir avec quelle race a eu lieu l'hybridation. La couleur de l'abeille noire française est donc toujours dans les classes I ou 2.

\section{b) L'index cubital}

Dans l'histogramme de la figure 3 les index cubitaux moyens des colonies sont groupés en classes de 0,04 . On constate que les index des ruches examinées, races étrangères exceptées, sont répartis entre $I, 46$ et $I, 89$ selon une distribution normale. L'index cubital moyen général, calculé à partir de toutes les colonies reconnues de race pure est de 1,70. Nous avons fait la discrimination entre les colonies de race noire, pour lesquelles on ne pouvait déceler aucune trace d'hybridation, les colonies hybrides et les colonies de races étrangères. Les hybridations entre races donnant des index cubitaux moyens d'égale valeur ne peuvent évidemment être décelées à l'aide de ce caractère. Lorsque l'index cubital d'une colonie est plus bas que l'index moyen de la race noire le risque d'hybridation avec une race étrangère à index différent est pratiquement nul, car il n'y a pas de races d'abeilles présentant un index cubital plus faible que la race noire. Par contre, le risque s'accroît rapidement dès que l'on dépasse l'index moyen de $\mathrm{I}, 7 \mathrm{O}$. Pour l'ensemble de nos analyses et en fontion du nombre de colonies dont les index sont groupés dans la même classe, le risque d'hybridation non décelable par l'index cubital est approximativement de:

7 p. Ioo dans la classe des index moyens de 1,74

I2 2 p. 100 - $\quad$ - $\quad$ I 78

I4 p. $100 \quad-\quad-\quad-\quad r \quad r, 82$

I6 p. IOO - $\quad-\quad$ I,S6

33 p. $100 \quad-\quad$ - $\quad I, 90$ 
Pour la classe des index moyens de $\mathrm{I}, 94$ on peut estimer que ce risque est d'environ 50 p. Ioo mais le nombre de ruches groupées dans cette classe est beaucoup trop faible pour permettre une estimation précise. A partir de la classe 1,98 on ne

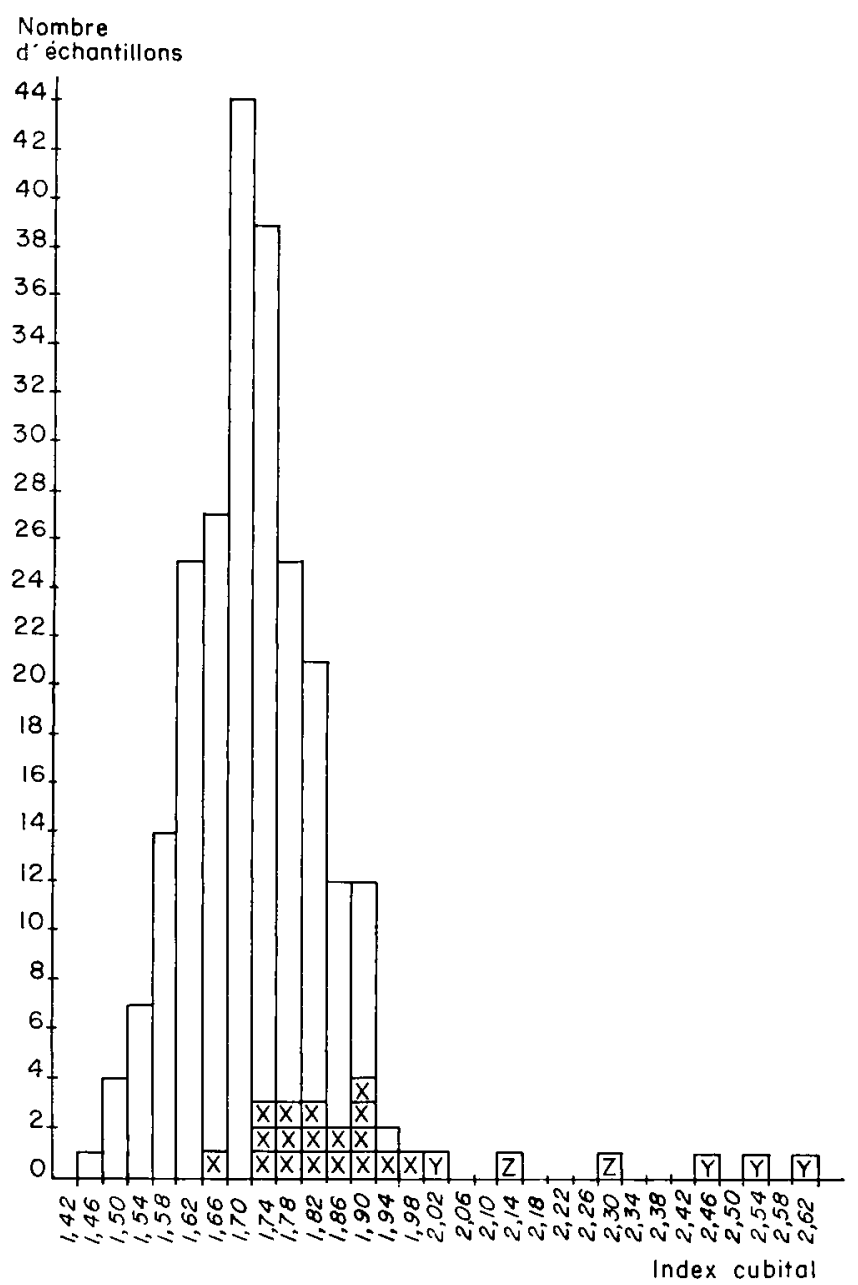

Fig. 3. - Histogramme de la répartition de l'index cubilal moyen à partir de 240 analyses, montrant la pénélration de l'index cubital de colonies hvbrides justu'à l'index cubital moyen général

\footnotetext{
$\square$ colonies d'abeilles Apis mellifica mellifica de race pure. $\bar{X}$ colonies d'abeilles hybrides.

Y colonies d'abeilles Apis mellifica ligustica de race pure.

$\overline{\mathbf{Z}}$ colonies d'abeilles Apis mellifica intermissa de race pure.
}

trouve plus d'abeilles de pure race noire et dans la classe 2,02 qui la suit on trouve la première colonie de race italienne pure.

On ne peut détailler ici les histogrammes de 240 analyses qui composent cette 


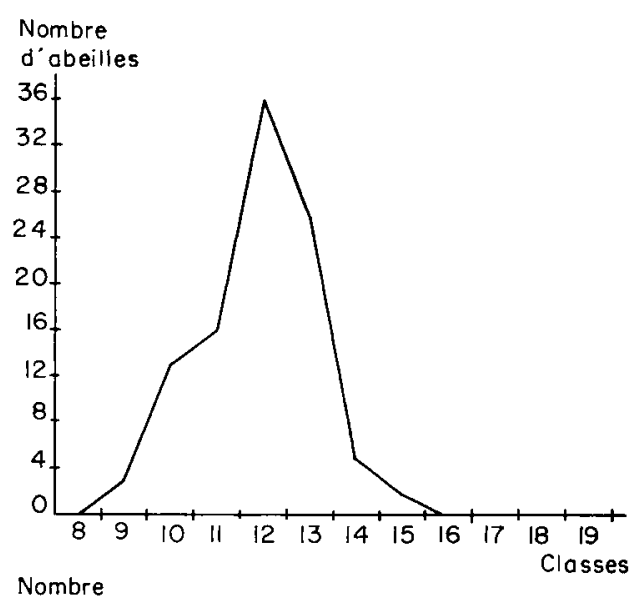

d'abeilles

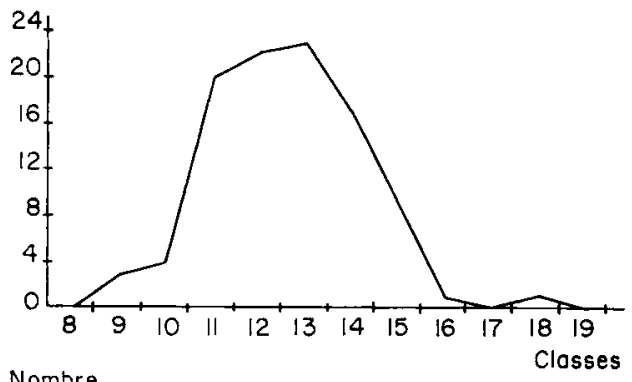

Nombre

Classes

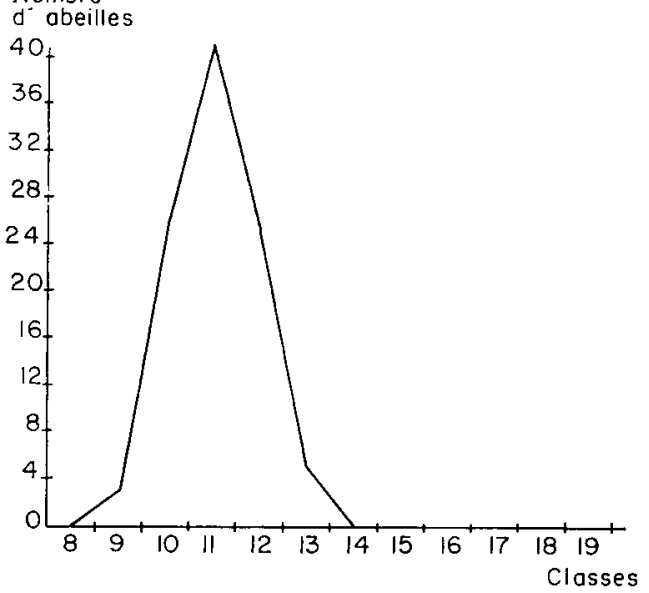

Fic: 4. - Itislogramme te Tindex cubital d'une colonie de pure race Apis mellifica mellitica donl les carnitéristiques sont très voisines des moyennes générales de notre élude

L'index cubital moyen est de $\mathrm{x}, 70$, l'écart-ty]e de $\mathrm{I}, 26$

FIG. 5. -- Hislogramme de l'index cubilal d'une colonie d'abeilles hybrides (Mellifica $\times$ Ligustica)

L’index cubital moyen est de I,8r, l'écart-type de I,59

lici. 6. - Histogramme de l'index cubital d'une colonie d'abeilles issue d'une lignée consanguine de pure race Apis mellifica mellifica

L'index cubital moyen est de 1,57 , l'écart-type de $0,9 \mathrm{I}$. 
étude. Quelques exemples suffiront à montrer les indications qu'ils donnent et leur relation avec l'écart-type.

Figure 4. - Histogramme d'une colonie dont les caractéristiques sont très

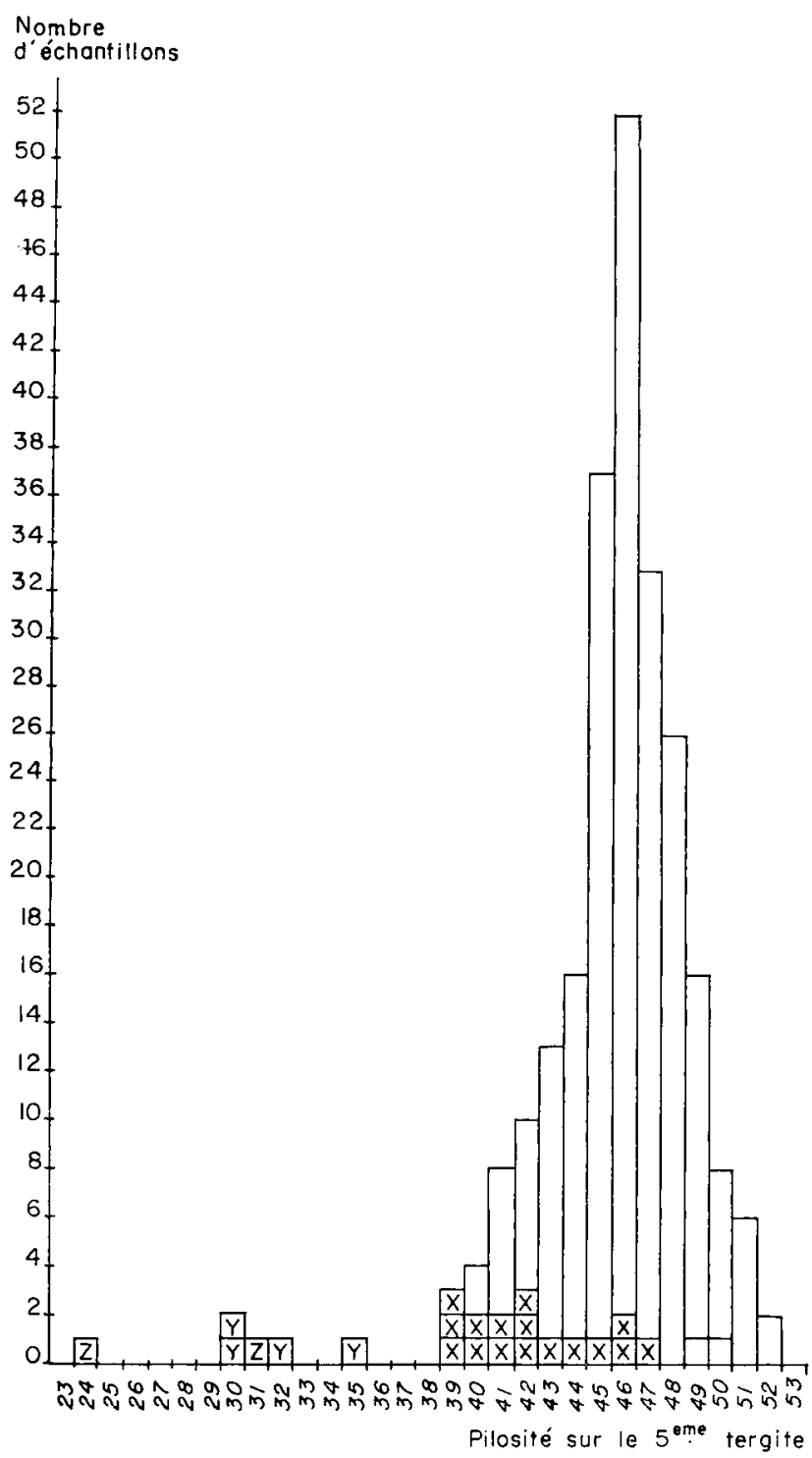

FIG. 7. - Hislogranme de la répartition de la longueur moyenne de la pilosité sur le cinquième tergite (2.0 analvises)

\footnotetext{
$\square$ colonies d'abeilles ipis mellifica mellifica de race pure.

$\bar{X}$ colonies d'abeilles hybrides.

$\bar{Y}$ colonies d'abeilles Apis mellifica ligustica de race pure.

$\overline{\mathbf{Z}}$ colonies dabeilles A pis mellifica intermissa de race pure.
} 
voisines des moyennes générales de notre étude. L'index cubital moyen est de I,70, l'écart-type de 1,26 .

Figure 5. - Histogramme d'une colonie hybride (Mellifica $\times$ Ligustica) dont l'hybridation remonte à 2 générations. L'index cubital moyen est de $\mathrm{I}, 8 \mathrm{I}$, on voit qu'il pourrait être celui d'une colonie de pure race noire. L'écart-type est de I,59 mais on ne peut cependant pas en conclure que la valeur de l'écart-type s'accroît toujours lors d'une hybridation car nous avons des exemples d'écart-type ne dépassant pas I,I9 pour des colonies ayant exactement le même degré d'hybridation que la

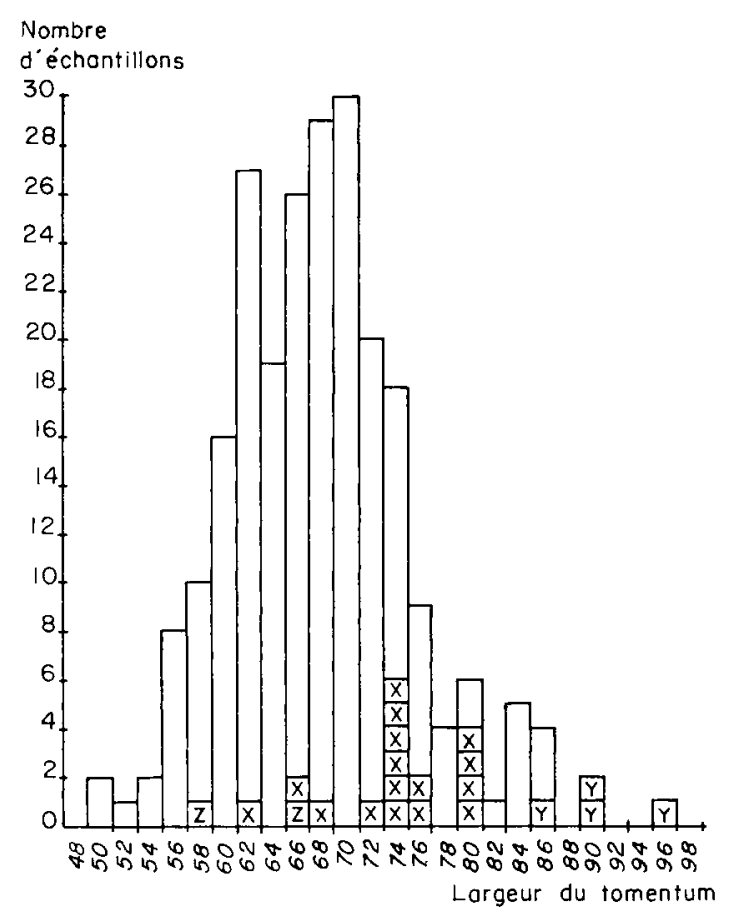

FIG. 8. - Histogramme de la répartition de la largeur moyenne_du lonientum (2 to analyses)

$\square$ colonies d'abeilles A pis mellifica mellifica de race pure.
$\frac{\square}{\overline{\mathrm{X}}}$ colonies d'abeilles hybrides.
$\overline{\mathrm{Y}}$ colonies d'abeilles Apis mellifica ligustica de race pure.
$\overline{\mathrm{Z}}$ colonies d'abeilles Apis mellifica intermissa de race pure.

précédente. La valeur de l'index cubital des 2 parents de races différentes infue directement sur l'écart-type des hybrides $\mathrm{F}_{1}$ et $\mathrm{F}_{2}$. Si, comparativement à leurs races respectives, l'index cubital du parent de race noire est élevé et l'index du parent de race italienne est faible, l'écart-type sera bas. Si les positions des parents sont inverses l'écart-type sera très élevé.

Figure 6. - Histogramme d'une colonie de pure race noire après 2 générations d'étroite consanguinité (frères $x$ sœurs). L'index cubital est de I,57. Exceptionnellement bas, il écarte toute possibilité d'hybridation avec des races à index élevé 
L'écart-type est de 0,9 I. Il est, lui aussi, particulièrement bas ce qui est un signe de l'homozygotie obtenue à la suite de l'élevage consanguin.

I a moyenne générale de l'écart-type de l'index cubital des colonies de pure race noire examinées dans nos analyses est de I,29. Leur amplitude va de 0,90 pour les plus faibles à I,80 pour les plus élevés.

\section{c) Pilosité du cinquième tergite abdominal}

Dans la figure 7 nous avons groupé les longueurs moyennes de la pilosité du $5^{\text {e }}$ tergite abdominal des colonies en classes de un dixième de millimètre. Nous avons également fait la discrimination entre les ruches de race noire, les hybrides et les colonies de races étrangères. Bien que la plupart des colonies se situent dans les limites assignées à la race noire, on trouve des exceptions dues aux hybridations. I a longueur moyenne de la pilosité du $5^{\mathrm{e}}$ tergite abdominal est de 0,46 pour l'ensemble des analyses des colonies de race noire. Flle se répartit entre 0,40 et 0,52 pour celle-ci.

\section{TABLEAU I}

Caractéristiques biométriques de quelques races d'abeilles susceptibles d'avoir été importées en France, comparćes avec les conclusions de notre étude sur la race Noire française

D'après les travaux de Giavarini (i953), Goetze (ig63), Louis (I963), Rihar (ig61), Ruttner et MACKENSEN (I954), RutTNer (I963).

\begin{tabular}{|c|c|c|c|c|c|}
\hline $\begin{array}{c}\text { Caractères } \rightarrow \\
\text { Races } \\
\downarrow\end{array}$ & Couleur & $\begin{array}{l}\text { Index } \\
\text { cubital }\end{array}$ & $\begin{array}{l}\text { Pilosite du } \\
5^{e} \text { tergite }\end{array}$ & $\begin{array}{l}\text { Tomentum } \\
\text { (index) }\end{array}$ & $\begin{array}{c}\text { Longueur de } \\
\text { la langue }\end{array}$ \\
\hline $\begin{array}{l}\text { Mellifica } \\
\text { (France) }\end{array}$ & $\begin{array}{c}\text { noire } \\
\text { petites taches } \\
\text { jaunes }\end{array}$ & $\begin{array}{c}1,70 \\
1,46-1,98\end{array}$ & $\begin{array}{c}0,,^{\prime} 6 \\
0,40-0,52\end{array}$ & $\begin{array}{c}0,91 \\
0,55-1,55\end{array}$ & $\begin{array}{c}6,35 \\
6,10-6,50\end{array}$ \\
\hline $\begin{array}{c}\text { Mellifica } \\
\text { (Allemagne) }\end{array}$ & $\begin{array}{c}\text { noire } \\
\text { petites taches } \\
\text { jaunes }\end{array}$ & $\begin{array}{c}1,80 \\
1,50-2,10\end{array}$ & $\begin{array}{c}0,50 \\
0, \mathbf{1} 0-0,60\end{array}$ & $\begin{array}{c}1,00 \\
0,50-1,80\end{array}$ & $\begin{array}{c}6,30 \\
6,00-6,40\end{array}$ \\
\hline $\begin{array}{l}\text { Ligustica } \\
\text { (Italie) }\end{array}$ & $\begin{array}{c}1 \text { à } 3 \\
\text { anneaux } \\
\text { jaunes }\end{array}$ & $\begin{array}{c}2,30 \\
2,00-2,70\end{array}$ & $\begin{array}{c}0,30 \\
0,20-0,40\end{array}$ & $\begin{array}{c}2,30 \\
1,50-2,80\end{array}$ & $\begin{array}{c}6,50 \\
6,30-6,60\end{array}$ \\
\hline $\begin{array}{c}\text { Carnica } \\
\text { (Autriche- } \\
\text { Yougoslavie) }\end{array}$ & $\begin{array}{c}\text { noire } \\
\text { taches jaunes } \\
\text { sur } 1 \text { ou } 2 \\
\text { anneaux }\end{array}$ & $\begin{array}{c}2,60 \\
2,3-3,00\end{array}$ & $\begin{array}{c}0,30 \\
0,20-0,40\end{array}$ & $\begin{array}{c}2,70 \\
1,50-3,00\end{array}$ & $\begin{array}{c}6,70 \\
6,40-6,80\end{array}$ \\
\hline $\begin{array}{c}\text { Cancasica } \\
\text { (Russie) }\end{array}$ & $\begin{array}{c}\text { noire } \\
\text { petites taches } \\
\text { jaunes }\end{array}$ & $\begin{array}{c}2,10 \\
1,90-2,30\end{array}$ & $\begin{array}{c}0,35 \\
0,30-0,40\end{array}$ & $\begin{array}{c}2,40 \\
1,80-2,90\end{array}$ & $\begin{array}{c}6,90 \\
6,70-7,00\end{array}$ \\
\hline $\begin{array}{l}\text { Intermissa }\left(^{1}\right) \\
\quad \text { (Maroc) }\end{array}$ & $\begin{array}{c}\text { noire } \\
\text { petites taches } \\
\text { jaunes }\end{array}$ & $\begin{array}{c}2,20 \\
2,10-2,30\end{array}$ & $\begin{array}{c}0,25 \\
0,20-0,35\end{array}$ & $\begin{array}{c}0,80 \\
0,75-1,00\end{array}$ & $\begin{array}{c}6,45 \\
6,30-6,60\end{array}$ \\
\hline
\end{tabular}

(1) A titre seulement indicatif, mesures pratiquées sur peu de colonies. 


\section{d) Largeur du tomentum}

La répartition des largeurs du tomentum est représentée dans la figure 8 . Les largeurs moyennes de chaque colonie sont groupées en classes de $0,05 \mathrm{~mm}$. De nombreuses colonies hybrides sont mélangées aux colonies noires de race pure. La largeur moyenne du tomentum pour ces dernières est de $0,67 \mathrm{~mm}$. Les largeurs extrêmes sont de $0,50 \mathrm{~mm}$ et $0,85 \mathrm{~mm}$.

\section{e) Longueur de la langue}

La longueur moyenne de la langue des colonies de pure race noire analysées est de 6,35 millimètres. Les plus courtes mesurent 6, Io mm, les plus longues 6,50 mm. Plus encore que pour les autres caractères les colonies hybrides sont mélangées aux colonies de race noire car la différence de longueur moyenne dans les races considérées est trop peu importante, à plus forte raison est-elle pour les hybrides, non discriminatoire.

Afin de permettre des comparaisons entre diverses races d'abeilles importées en France ou susceptibles de l'être et l'abeille indigène, nous avons groupé dans le tableau I les résultats publiés par divers auteurs.

\section{DISCUSSION}

I,es nombreuses analyses biométriques que nous avons réalisées sur des colonies de pure race noire nous permettent de définir quelques caractéristiques importantes de l'abeille noire française que l'on peut résumer de la façon suivante :

1) Couleur... Noire, avec de très petites taches jaunes sur le $1^{\text {er }}$ tergite.

2) Index cubital ..................... moyenne 1,70 amplitude $1,46-1,98$

3) Pilosité du 5 e tergite $\ldots \ldots \ldots \ldots \ldots \ldots \ldots$ moyenne 0,46 amplitude $0,40-0,52$

4) Largeur du tomentum $\ldots \ldots \ldots \ldots \ldots \ldots \ldots$ moyenne 0,67 amplitude $0,50-0,85$ (Index tomentum) .................. moyenne 0,91 amplitude $0,55-1,55$

5) Longueur de la langue $\ldots \ldots \ldots \ldots \ldots \ldots$ moyenne 6,35 amplitude $6,10-6,50$

Ces moyennes montrent la parfaite identification de la race noire française avec l'abeille noire d'Europe décrite par GOETZE et par RUTTNER, et prouvent qu'il s'agit bien de la même race d'abeille (tab1. I). Les variations des mesures biométriques sont plus importantes au sein des études de chaque auteur qu'entre les moyennes obtenues par ceux-ci. Ces légères différences peuvent d'ailleurs être en partie imputées aux méthodes de travail et aux utilisateurs. La variabilité dans chaque groupe étudié est suffisamment importante pour rendre impossible la discrimination entre des colonies appartenant à l'un ou l'autre de ces groupes.

Il est possible que les moyennes des mesures biométriques faites en France puissent présenter localement de légères variantes à caractère régional qui pourraient être en corrélation avec l'adaptation, déjà reconnue, au climat et à la flore. Cependant it s'agirait de variations de faible amplitude, seulement significatives sur les moyennes et la possibilité de détection de colonies appartenant à ces divers " écotypes " est encore plus improbable que dans le cas de colonies venant de différents 
pays. Fin outre, nous assistons depuis de nombreuses années à un brassage intense des abeilles de diverses régions de France dû̀ à la transhumance des ruches. Les multiples origines des colonies de la Station expérimentale d'Apiculture nous permettent en conséquence de considérer notre cheptel comme représentatif de l'aspect morphologique de l'abeille noire française.

L'étude d'analyses biométriques de colonies de races pures, d'hybrides, la comparaison avec les travaux antérieurs, démontrent qu'il est parfois extrêmement difficile de formuler un diagnostic sûr et précis. Dans certains cas on ne pourra lever le doute sur une hybridation possible. Pour chacun des caractères morphologiques la variation naturelle est importante, les mesures extrêmes possibles pour chaque race se confondent avec les extrêmes d'une ou plusieurs autres races. Les risques d'hybridation apparaissent, ainsi que nous l'avons montré, dès que l'on s'écarte de la moyenne définie pour un caractère, et non seulement pour les données proches des limites de 1'amplitude.

Ces considérations démontrent aussi la nécessité absolue de la pluralité et de la diversité des mesures dans les analyses biométriques.

Reçu pour publication en septembre 196.5 .

\section{SUMMARY}

A BIOMETRIC STUDY OF SOME MORPHOLOGICAL CHARACTERISTICS

OF THE FRENCH BLACK BEE ("APIS MELLIFICA MELLIFICA")

The author describes the average biometric characteristics of the indigenous French bee (Apis mellifica mellifica). The colour is black, sometimes with small yellow spots on the first abdominal tergite, the cubital index is 1.70 , the pilosity of the fifth tergite is $0.46 \mathrm{~mm}$, the tomentum index is $0.9 \mathrm{I}$, the tongue-length is $6.35 \mathrm{~mm}$. The diagnostic difficulties in the biometric analyses of certain hybrid colonies are also demonstrated.

\section{RÉFÉRENCES BIBLIOGRAPHIQUES}

DREHER K., I950. Beiträge zur Merkmalsuntersuchung der Honigbiene. Z. Bienentorsch., 1, (2), I 7-23. Gravarini I., I953. Ricerche sui caratteri razziali dell' Apis mellifica ligustica spinola. Estr. Mem. Soc. Entomol. Ital., 32, I I9-1 28.

Goetze G., I963. Die Honigbiene in natürlicher und Künstlicher Zuchtaüslesc. Paul Parey, IHambourg, 212 pages.

LouIs J., 1963. Étude de la translation du point discoüdal (Discoüdalverschiebung) de l'aile de l'abeille (Apis mellifica L.). Ann. Abeille, 6, 303-320.

Rihar J., г96. Recherches biométriques sur la couleur des abeilles carniolicmes en Yougoslavie. (Apis mellifica camica Pollm.) Ann. Abeille, 4, 4I-50.

Ruttner F., Mackensen O., I954. The genetics of the honeybee. Bee World, 33, 53-62, $71-79$.

Ruttner F., ig63. Die Zuchtauslese bei der Biene. N-O. Imkerschule Wr Neustadt. Walthergasse, 6. 79 pages. 